\title{
Adaptação e validade de conteúdo da Prova de Consciência Fonológica para Moçambique
}

\author{
Gérson O. E. Muitana' e Cibelle A. de la H. Amato² \\ 1 Departamento de Psicologia, Universidade Eduardo Mondlane (UEM) \\ 2 Programa de Pós-Graduação em Distúrbios do Desenvolvimento, \\ Universidade Presbiteriana Mackenzie (UPM)
}

Submissão: 15 set. 2020 .

Aceite: 17 maio 2021.

\section{Nota dos autores}

Gérson O. E. Muitana (D) https://orcid.org/0ooo-00o2-0724-6607

Cibelle A. de la H. Amato (D) https://orcid.org/00oo-0003-2422-6998

Financiamento: Programa de Excelência Acadêmica - Proex (1133/2019).

Correspondências referentes a este artigo devem ser enviadas para Cibelle A. de la H. Amato, Programa de Pós-Graduação em Distúrbios do Desenvolvimento, Centro de Ciências Biológicas e da Saúde, Universidade Presbiteriana Mackenzie (UPM), Rua da Consolação, 930, Prédio $28,1^{\circ}$ andar, São Paulo, SP, Brasil. CEP 01302-907.E-mail: cibelle.amato@mackenzie.br 


\title{
Resumo
}

A consciência fonológica é uma habilidade muito importante para o desempenho em leitura, escrita e matemática. Sugere-se que seja monitorada e avaliada preferencialmente durante a pré-escola como forma de intervenção precoce para prevenção de dificuldades de aprendizagem após o ensino formal. Contudo, para sua avaliação, é necessário ter instrumentos qualificados, e, em Moçambique, não existe nenhum que avalie essa habilidade. Portanto, este estudo teve o objetivo de adaptar culturalmente a Prova de Consciência Fonológica por produção Oral (PCFO) para o contexto moçambicano, o que foi realizado em duas fases: equivalência semântica e validação de conteúdo. O Índice de Validade de Conteúdo foi de 0,96; embora seja considerado ótimo, estudos posteriores precisam ser realizados para verificar outros parâmetros de validade, fidedignidade e normatização. Espera-se que o instrumento contribua para avaliação da consciência fonológica e que, acima de tudo, a habilidade faça parte das atividades de estimulação e intervenção em pré-escolares.

Palavras-chave: adaptação cultural, validade de conteúdo, consciência fonológica, Moçambique, avaliação

\section{ADAPTATION AND CONTENT VALIDITY OF THE PHONOLOGICAL AWARENESS TEST FOR MOZAMBIQUE}

\begin{abstract}
Phonological awareness is an essential skill for performance in reading, writing, and mathematics. Therefore, it is suggested that it be monitored and evaluated preferably during preschool as a form of early intervention to prevent learning difficulties after formal education. However, it is necessary to have qualified instruments for its evaluation, and in Mozambique, none assesses this skill. Therefore, this study aimed to culturally adapt the Phonological Awareness Test by Oral production (PATOP) to the Mozambican context and was carried out in two phases: semantic equivalence and content validation. The Content Validity Index (CVI) was 0.96; although it is considered excellent, further studies need to be carried out to verify other validity, reliability, and standardization parameters. Nevertheless, it is expected that the instrument contributes to the evaluation of phonological awareness and that, above all, the skill is part of activities of stimulation and intervention in preschoolers.

Keywords: cultural adaptation, content validity, phonological awareness, Mozambique, evaluation
\end{abstract}

\section{ADAPTACIÓN Y VALIDEZ DE CONTENIDO DE PRUEBA DE CONCIENCIA FONOLÓGICA PARA MOZAMBIQUE}

\section{Resumen}

La conciencia fonológica es una habilidad muy importante para el desempeño en lectura, escritura y matemáticas, lo que sugiere que se monitorea y evalúa preferiblemente en preescolar como una forma de intervención temprana para prevenir dificultades de aprendizaje en la educación formal. Sin embargo, para su evaluación es necesario contar con instrumentos calificados y en Mozambique no hay 
ninguno que evalúe esta habilidad. Por lo tanto, este estudio tuvo como objetivo adaptar culturalmente el Test de Conciencia Fonológica por producción Oral para Mozambique y se llevó a cabo en dos fases: equivalencia semántica y validación de contenido. El Índice de Validez de Contenido fue de 0,96, aunque se considera excelente, es necesario realizar más estudios para verificar otros parámetros de validez, confiabilidad y estandarización. Se espera que el instrumento contribuya a la evaluación de la conciencia fonológica y que sobre todo, la habilidad sea parte de las actividades de estimulación e intervención en preescolares.

Palabras clave: adaptación cultural, validez de contenido, conciencia fonológica, Mozambique, evaluación 
A consciência fonológica (CF) é uma habilidade que permite refletir sobre os segmentos sonoros do que falamos, ou seja, que a língua falada pode ser segmentada em pequenas unidades, a frase em palavras, as palavras em sílabas e as sílabas em fonemas. Consiste, portanto, em detectar e manipular sons, sílabas e palavras como seu subconjunto (Ciesielski \& Creaghead, 2020). Desenvolve-se durante a pré-escola e nos primeiros anos escolares, progredindo em diferentes unidades linguísticas: da consciência de palavras e sílabas à consciência de unidades intrassilábicas - como onset e rima - e, finalmente, à consciência de fonema (Goswami \& Bryant, 1900).

A CF é muito importante para o desempenho acadêmico e é descrita por The National Early Literacy Panel (Nelp) como uma das seis habilidades e competências infantis que melhor predizem o desempenho posterior em leitura e escrita (Shanahan \& Lonigan 2015). Além disso, estudos mostram que essa consciência se correlaciona significativamente com a precisão e fluência de leitura, podendo prevê-las nos alunos da primeira à terceira série do ensino fundamental (Song et al., 2015; Vibulpatanavong \& Evans, 2019). Em crianças disléxicas, a CF é a habilidade com maior déficit desde os períodos pré-escolar e escolar (Snowling \& Melby-Lervåg, 2016). Outros resultados têm mostrado a existência de correlação positiva e significativa entre a CF e a escrita (Santos et al., 2017), e com a competência aritmética em crianças do primeiro ano, antecipando o desempenho matemático em crianças do ensino fundamental (Pazeto et al., 2020; Pinto et al., 2016).

Para além das correlações apresentadas, os programas de intervenção e estimulação em CF têm demonstrado sua eficácia na promoção de benefícios para o desempenho da escrita e da compreensão leitora em crianças disléxicas (Germano et al., 2013; Pape-neumann et al., 2015). Ademais, têm evidenciado ganhos de longo prazo em leitura para crianças de jardim de infância (Diamanti et al., 2017), assim como benefícios em leitura, decodificação e compreensão de palavras em crianças consideradas de risco e atípicas (Fälth et al., 2017; Kjeldsen et al., 2019)

Por conta da sua importância para o desempenho acadêmico, uma das estratégias de intervenção e prevenção precoce, para além da estimulação da habilidade, tem sido avaliar crianças nos anos iniciais de alfabetização para identificar aquelas que podem estar em risco de apresentar dificuldades ou transtorno específico de aprendizagem futuramente. No entanto, em realidades como Moçambique, onde não há instrumentos de avaliação, uma das recomendações tem sido realizar adaptação cultural, por ser vantajosa em relação a custos (de tempo e financeiros) e possibilitar intercâmbios internacionais de investigação científica, quando se compara isso com o desenvolvimento de um novo instrumento (Hambleton, 2005).

Assim, olhando para a realidade brasileira por exemplo, em que a produção científica está mais desenvolvida em relação a Moçambique nessa temática, foi possível mapear instrumentos existentes naquele país, tendo sido verificado que a Prova de Consciência Fonológica 
por produção Oral (PCFO) possuía ótimas propriedades psicométricas, dados normativos e consistência nos seus resultados. Considerando a existência de uma proximidade histórica, cultural e de idioma (Morais, 2019), além do fato de que as duas variedades linguísticas compartilham o mesmo léxico básico (Petter, 2008, 2009), esses aspectos contribuíram bastante como critérios para que se diminuíssem etapas para a obtenção do instrumento e que a PCFO fosse a escolhida para adaptação.

A PCFO é um instrumento que avalia dez componentes da CF, envolvendo a manipulação de sons da fala (Capovilla \& Capovilla, 1998). Estudos com o instrumento têm sido conduzidos nos últimos 14 anos (Dias et al., 2013). Capovilla (2006, como citado em Dias et al., 2013) investigou dados de fidedignidade numa amostra de 379 crianças brasileiras da primeira à quarta série (do primeiro ao quarto ano em Portugal ou da primeira à quarta classe em Moçambique) e obteve os seguintes resultados: alfa de Cronbach de 0,91, coeficiente de Spearman-Brown de 0,86 e teste-reteste com coeficiente de 0,87. Esses resultados foram considerados muito satisfatórios. Para a consistência interna, Capovilla et al. (2007) encontraram correlações positivas e significativas, com magnitudes de moderada a alta, entre cada um dos dez subtestes da prova e o desempenho total no instrumento em crianças de 6 a 15 anos de idade. $O$ estudo revelou que a prova apresentava evidências de validade para avaliar CF em crianças, especificamente da primeira à terceira série. A prova também apresenta dados normativos para crianças de escolas públicas já a partir de 3 anos de idade (Dias et al., 2013) e normas para crianças do pré 1 à segunda série de escola particular (Seabra \& Capovilla, 2011).

De forma geral, os estudos demostraram evidências de validade por correlação com outras variáveis com uso de técnicas diversificadas, como observação de mudanças com o desenvolvimento; correlação com outros testes, revelando padrões de convergência com construtos correlatos; e correlação com critérios externos, a exemplo da nota escolar (Dias et al., 2013), para além de estudos em crianças e adolescentes com síndrome de Williams-Beuren e síndrome de Down. Isso demostra que o instrumento possui características psicométricas adequadas para avaliação de crianças e adolescentes brasileiros, da educação infantil ao fundamental.

Portanto, é um instrumento que será muito importante para o contexto moçambicano em que o ensino pré-escolar, para além de ser facultativo, é assegurado por instituições privadas ou comunitárias (Timbane, 2016), fato que tem contribuído para uma diversificação de conteúdos e não sistematização de currículos utilizados em creches, jardins de infância e centros infantis. Além disso, cada instituição infantil é livre para criar e adaptar os materiais, trazendo experiências de outras realidades, e por vezes a produção desse material pode ficar aquém do pretendido (Francisco, 2015). Contudo, essa ferramenta será extremamente fundamental, visto que alguns estudos têm apontado que crianças moçambicanas apresentam déficits de competências básicas de leitura e escrita da língua portuguesa; déficits de reconheci- 
mento de letras do alfabeto; e déficit em competências de contagem e cálculo (Fundação Aga Khan, 2011; TPC Moçambique, 2017). Outros estudos concluíram que uma das causas dos déficits têm sido a preparação inadequada das crianças e a penúria de pré-requisitos destas antes da sua entrada no ensino primário (Nhampossa, 2018; Organização das Nações Unidas para a Educação, a Ciência e a Cultura [Unesco], 2007; United Nations Educational, Scientific and Cultural Organizational [Unesco], 2012). Desse modo, um instrumento de avaliação de CF pode contribuir para que crianças sejam avaliadas principalmente na pré-escola e sejam encaminhadas precocemente para prevenir possíveis dificuldades de aprendizagem (DA) no ensino formal. Além disso, fornecerá oportunidades educativas adequadas e consequentemente reduzirá as altas taxas de inconclusão do ensino primário, assim como as altas taxas de reprovações naquele país africano (Unesco, 2007, 2012).

Em relação ao processo de adaptação cultural de instrumentos psicológicos de um determinado contexto para o outro, Fonseca et al. (2011) referem que ele não se resume apenas à tradução (porque o idioma e a cultura interferem no processo de avaliação como um todo). Sendo assim, salienta-se que a adaptação siga procedimentos, etapas e técnicas específicos para além de que obedeça a um certo rigor metodológico. Uma das primeiras etapas nesse processo é a busca por evidências de validade de conteúdo que procura verificar se, quando adaptado, o instrumento continua mensurando o mesmo construto (Pasquali, 2013). Essa busca é verificada por meio da análise e do julgamento dos especialistas (Pasquali, 2003), e, para isso, podem ser usadas pelo menos duas abordagens, geralmente qualitativa e quantitativa. A qualitativa é realizada por meio de equivalências semântica, idiomática, conceitual e experimental (Guillemin, 1995), já uma das formas para a abordagem quantitativa tem sido por meio do cálculo do Índice de Validade de Conteúdo (IVC), para quantificar o grau de concordância entre os especialistas (Coluci et al., 2015).

Portanto, este estudo tem o objetivo de realizar a adaptação cultural e validação de conteúdo da PCFO para o contexto moçambicano. Acredita-se que esse instrumento será útil na medida em que será o primeiro no país, esperando-se que contribua grandemente para avaliação neuropsicológica, principalmente no rastreio de preditores de desempenho acadêmico. Aspira-se também a que mais estudos sejam realizados com o instrumento e que futuramente sejam verificadas outras evidências de validade e precisão, para além de serem elaborados dados normativos para o seu uso na clínica e em pesquisas científicas.

\section{Método}

Trata-se de um estudo metodológico de adaptação cultural de um instrumento. Todos os procedimentos metodológicos e todas as abordagens teóricas usados basearam-se em pesquisas científicas sobre o tema e nas diretrizes do International Test Commission (ITC) para tradução e adaptação de testes (segunda edição). O estudo foi realizado em duas fases: 1 - 
equivalência semântica realizada por nativos moçambicanos e 2 - validação de conteúdo realizada por um comitê de juízes e especialistas.

\section{Participantes}

Na fase 1, inicialmente foram convidados dez indivíduos, mas participaram do estudo nove nativos, adultos, universitários e moradores da cidade de São Paulo há dois anos em média $(D P=2,2)$, sendo seis do sexo masculino e três do feminino, de 26 a 47 anos. Um dos critérios usados para a seleção foi a assinatura do Termo de Consentimento Livre e Esclarecido (TCLE) e ter vivido em Moçambique por pelo menos dez anos. Na fase 2, foram convidados seis juízes, mas participaram quatro, sendo eles: dois psicólogos com especialização em Desenvolvimento e Educação de Infância, um mestre em Avaliação Psicológica e uma pedagoga especializada em Psicologia e Intervenção Infantil. Os critérios para seleção foram: ter no mínimo o título de mestre ou especialização preferencialmente na área de avaliação psicológica infantil e ter pelo menos cinco anos de experiência prática nessas áreas.

\section{Processo de adaptação cultural}

Por questões éticas de pesquisa, primeiro se obteve o consentimento formal dos autores do instrumento para autorização da adaptação, e, a seguir, o projeto foi aprovado pelo Comitê de Ética da Universidade Presbiteriana Mackenzie (UPM) - Certificado de Apresentação para Apreciação Ética (CAAE) nº 15548519.4.0000.0084. Todos os indivíduos foram convidados a participar espontaneamente, mediante a assinatura do TCLE.

Na fase 1, o instrumento foi submetido a uma análise equilibrada de considerações linguísticas, culturais e contextuais por parte do estrato da população-alvo. Avaliou-se e analisou-se a adequação de todos os itens do instrumento (palavras, frase e expressões) por meio dos critérios: bom, regular ou péssimo. Caso algum item estivesse inadequado ou incompreensível, solicitava-se aos nativos que apresentassem possíveis sugestões de mudança, que depois foram analisadas e alteradas no instrumento, para que fosse elaborada a PCFO: versão moçambicana preliminar. A seguir, na fase 2, o instrumento foi analisado por juízes moçambicanos para validação do conteúdo. Os especialistas verificaram se a versão adaptada refletia o conteúdo do item "clareza" conforme propõe a versão original, sugerindo alterações e recomendações, sendo depois elaborada a versão final (PCFO - versão moçambicana).

\section{Instrumentos}

\section{Prova de Consciência Fonológica por produção Oral}

A PCFO foi desenvolvida por Capovilla e Capovilla (1998), baseada no Teste de CF e no Teste Sound Linkage (Capovilla \& Capovilla, 1998). O teste é guiado pelo aplicador, que explica o que precisa ser feito, dando a instrução de forma oral, e, após isso, o examinando também 
dá a resposta de forma oral, e esta é anotada. A pontuação varia de 0 a 40, e cada resposta correta vale 1 ponto. A aplicação deve ser feita individualmente, e o tempo médio é de 20 minutos. Dez componentes da CF são avaliados por dez subtestes, cada um composto por quatro itens e dois exemplos iniciais (Seabra \& Capovilla, 2012). Os subtestes são:

1. Síntese Silábica: o aplicador pronuncia as sílabas de uma palavra dando o intervalo de um segundo entre elas, e o examinando deve uni-las dizendo qual palavra se dá com o resultado.

2. Síntese Fonêmica: o aplicador pronuncia separadamente os fonemas de uma palavra, e o examinando deve uni-los dizendo a palavra resultante da união.

3. Rima: o aplicador pronuncia três palavras, e o examinando deve selecionar entre elas as duas que terminam com o mesmo som.

4. Aliteração: o aplicador apresenta três palavras, e o examinando deve selecionar entre elas as duas que começam com o mesmo som.

5. Segmentação Silábica: o aplicador pronuncia uma palavra, e o examinando deve repeti-la separando-a em suas sílabas componentes.

6. Segmentação Fonêmica: o aplicador pronuncia uma palavra, e o examinando deve repeti-la em seus fonemas componentes.

7. Manipulação Silábica: o examinando deve adicionar e subtrair sílabas de palavras, dizendo qual a palavra formada, conforme a sílaba dada pelo aplicador.

8. Manipulação Fonêmica: o examinado deve adicionar e subtrair fonemas de palavras, dizendo qual a palavra formada conforme os fonemas fornecidos pelo aplicador.

9. Transposição Silábica: o aplicador dá uma palavra, e o examinando deve inverter a ordem das sílabas dizendo qual a palavra nova formada.

10. Transposição Fonêmica: o examinando deve inverter a ordem dos fonemas de palavras dizendo qual a palavra nova formada.

\section{Questionário de equivalência semântica para nativos}

Foi desenvolvido para este estudo e tinha por objetivo coletar informações de nativos em relação à sua percepção sobre termos e expressões da prova original que não seriam comumente usados em Moçambique. Além de coletar dados demográficos, apresentava uma tabela com todos os itens dos dez subtestes da PCFO (descrição da tarefa, instruções, treinos 1 e 2 , e testes 1, 2, 3 e 4) e das duas seções (dados da prova e estrutura/apresentação), seguidos de espaços em branco para assinalar a avaliação (bom, regular ou péssimo). Caso a avaliação fosse regular ou péssima, os nativos sugeriam alterações considerando as características do item original. 


\section{Questionário para juízes e especialistas}

Foi desenvolvido para este estudo e criado a partir da plataforma on-line Google Formulários, inspirado no modelo de Coluci et al. (2015). Na primeira parte, apresentavam-se o TCLE e a declaração dos pesquisadores. Na segunda, era solicitado aos juízes que avaliassem a clareza de todos os itens do instrumento e um item adicional (estrutura e apresentação) com base em três critérios: compreensão, objetividade, contextualização (linguística, semântica e idiomática). A compreensão avaliava a inteligibilidade de todos os itens do instrumento para o público-alvo. A objetividade avaliava a descrição adequada dos itens para sua correta aplicação e pontuação pelos aplicadores. A contextualização avaliava se palavras e expressões do instrumento apresentavam linguagem, vocabulário, significados e escrita comumente usados na população-alvo, enquanto a estrutura e apresentação avaliavam se todo o instrumento e a folha de respostas apresentavam uma organização adequada de texto, forma e estrutura. Os critérios eram avaliados e preenchidos de acordo com uma escala Likert que variava de 1 a 4 . Nessa avaliação, era solicitado aos juízes que também efetuassem mudanças, porém mantendo as propriedades do instrumento original.

\section{Análise dos dados}

Na fase 1, todos os dados foram analisados qualitativamente, considerando as sugestões de frases e expressões realizadas pelos nativos. Na fase 2 , foram analisados qualitativamente por meio de uma escala Likert ( 1 = não claro, 2 = pouco claro, 3 = bastante claro e $4=$ muito claro) (Pasquali, 2010). Todos os itens que tiveram pontuação de 1 e 2 e para os quais os juízes sugeriram alterações foram considerados como inadequados, depois analisados e alterados no instrumento. A seguir, os dados foram analisados quantitativamente, calculando-se o IVC dos itens (descrição da tarefa, instruções, treinos 1 e 2, e testes 1, 2, 3 e 4), dos dez subtestes e das duas seções (dados da prova e estrutura/apresentação da prova). Para calcular o IVC dos itens segundo os critérios propostos, somaram-se as respostas 3 e 4 dos juízes nos itens de cada subteste e dividiu-se o resultado dessa soma pelo número total de respostas, segundo a fórmula: IVC $=\mathrm{n}^{\circ}$ de respostas 3 ou $4 / \mathrm{n}^{\circ}$ total de respostas (Coluci et al., 2015; Grant \& Davis, 1997), e considerou-se aceitável e adequado um valor de IVC > 0,80 (Pasquali, 2010; Wynd et al., 2003). Para calcular o IVC total do instrumento, somaram-se todos IVC de cada critério dos dez subtestes e das duas seções, achando as suas médias aritméticas. Posteriormente, as médias aritméticas foram somadas e divididas pelo número de seções avaliadas em todo instrumento. Todas as sugestões e comentários dos juízes foram analisados e alterados. 


\section{Resultados}

$\mathrm{Na}$ fase 1 , os nativos avaliaram o instrumento e sugeriram alterações de palavras e construções frásicas para que fossem adequadas para o português falado em Moçambique, e depois foram realizadas alterações. A Tabela 1 apresenta as adaptações realizadas por nativos a partir da PCFO original.

\section{Tabela 1}

Adaptações realizadas por nativos a partir da PCFO original

\begin{tabular}{|c|c|c|c|c|}
\hline Prova & Item & $\begin{array}{c}\text { Palavra/expressão } \\
\text { da PCFO original }\end{array}$ & $\mathbf{N}$ & $\begin{array}{c}\text { Adaptação } \\
\text { realizada }\end{array}$ \\
\hline \multirow{3}{*}{ Dados da prova } & \multirow{3}{*}{$\begin{array}{l}\text { Instruções, } \\
\text { aplicação, pontuação }\end{array}$} & Registro & 9 & Registo \\
\hline & & Probando & 8 & Avaliado \\
\hline & & Correta & 9 & Correcta \\
\hline Síntese Silábica & Instrução & Está falando & 7 & Está a falar \\
\hline \multirow{4}{*}{ Rima } & \multirow[b]{2}{*}{ Treino 2} & Sereia & \multirow[b]{2}{*}{4} & Areia \\
\hline & & Canoa & & Coroa \\
\hline & Treino 3 & Rolha & & Folha \\
\hline & Treino 4 & Sopé & 0 & Maré \\
\hline \multirow{2}{*}{ Aliteração } & Treino 1 & Fada & 4 & Fama \\
\hline & Teste 1 & Boné & 6 & Bota \\
\hline \multirow{3}{*}{ Segmentação Silábica } & Treino 2 & Bexiga & 6 & Cidade \\
\hline & Testes 3 & Fazenda & 9 & Machamba \\
\hline & Testes 4 & Gelatina & 4 & Televisão \\
\hline \multirow{2}{*}{ Manipulação Silábica } & \multirow{2}{*}{ Instrução } & Adicionar & \multirow{2}{*}{5} & Acrescentar \\
\hline & & Subtrair & & Tirar \\
\hline
\end{tabular}

Nota. Dados retirados do questionário de equivalência semântica dos nativos.

Todas as palavras substituídas na prova tiveram como base o número de sílabas que pertencia à palavra original, e não necessariamente o grupo semântico, com o objetivo de deixar a versão adaptada mais próxima da versão original. Na fase 2, após a avaliação e pontuação dos quatro especialistas, foi calculado o IVC de cada seção do instrumento segundo os três critérios. A Tabela 2 apresenta o IVC de todas as seções da PCFO. Todos os valores dos critérios tiveram pontuações acima de 0,90, exceto o critério "contextualização" da seção "dados da prova, instruções, aplicação, pontuação" que teve pontuação de 0,75. 


\section{Tabela 2}

Índice de Validade de Conteúdo (IVC) de todas as seções da PCFO

\begin{tabular}{|c|c|c|c|c|c|c|c|c|c|c|c|c|}
\hline \multicolumn{13}{|c|}{ Dados da prova, instruções para aplicação e pontuação } \\
\hline \multirow{2}{*}{ Itens da prova } & \multicolumn{4}{|c|}{ Compreensão } & \multicolumn{4}{|c|}{ Objetividade } & \multicolumn{4}{|c|}{ Contextualização } \\
\hline & Não & Pouco & Bastante & Muito & Não & Pouco & Bastante & Muito & Não & Pouco & Bastante & Muito \\
\hline $\begin{array}{l}\text { Dados da prova, instruções, } \\
\text { aplicação, pontuação }\end{array}$ & 0 & 0 & 2 & 2 & 0 & 0 & 1 & 3 & 0 & 1 & 1 & 2 \\
\hline IVC & \multicolumn{4}{|c|}{1} & \multicolumn{4}{|c|}{1} & \multicolumn{4}{|c|}{0,75} \\
\hline \multicolumn{13}{|c|}{ Subteste Síntese Silábica } \\
\hline \multirow{2}{*}{ Síntese Silábica } & \multicolumn{4}{|c|}{ Compreensão } & \multicolumn{4}{|c|}{ Objetividade } & \multicolumn{4}{|c|}{ Contextualização } \\
\hline & Não & Pouco & Bastante & Muito & Não & Pouco & Bastante & Muito & Não & Pouco & Bastante & Muito \\
\hline Descrição & 0 & 0 & 2 & 2 & 0 & $\mathrm{O}$ & 2 & 2 & 0 & 1 & 1 & 2 \\
\hline Instruções & $\mathrm{O}$ & $\mathrm{O}$ & 2 & 2 & 0 & 0 & 3 & 1 & 0 & 0 & 1 & 3 \\
\hline Treino 1 & 0 & 0 & 0 & 4 & 0 & 0 & 0 & 4 & 0 & $\mathrm{o}$ & 0 & 4 \\
\hline Treino 2 & $\mathrm{O}$ & $\mathrm{O}$ & 0 & 4 & 0 & $\mathrm{O}$ & 0 & 4 & 0 & $\mathrm{o}$ & 0 & 4 \\
\hline Teste 1 & 0 & $\mathrm{O}$ & 0 & 4 & 0 & 0 & 0 & 4 & $\mathrm{O}$ & $\mathrm{O}$ & 2 & 2 \\
\hline Teste 2 & o & 0 & $\mathrm{O}$ & 4 & 0 & $\mathrm{o}$ & 0 & 4 & 0 & 0 & $\mathrm{O}$ & 4 \\
\hline Teste 3 & 0 & $\mathrm{O}$ & 0 & 4 & 0 & 0 & 0 & 4 & 0 & 0 & 1 & 3 \\
\hline $\begin{array}{l}\text { Teste } 4 \\
\text { IVC }\end{array}$ & \multicolumn{4}{|c|}{1} & \multicolumn{4}{|c|}{1} & \multicolumn{4}{|c|}{0,96} \\
\hline \multicolumn{13}{|c|}{ Subteste Síntese Fonêmica } \\
\hline \multirow{2}{*}{ Síntese Fonêmica } & \multicolumn{4}{|c|}{ Compreensão } & \multicolumn{4}{|c|}{ Objetividade } & \multicolumn{4}{|c|}{ Contextualização } \\
\hline & Não & Pouco & Bastante & Muito & Não & Pouco & Bastante & Muito & Não & Pouco & Bastante & Muito \\
\hline Descrição & 0 & 0 & 1 & 3 & 0 & 0 & 1 & 3 & 0 & 1 & 0 & 3 \\
\hline Instruções & $\mathrm{O}$ & $\mathrm{O}$ & 0 & 4 & 0 & 0 & 1 & 3 & $\mathrm{O}$ & 0 & 2 & 2 \\
\hline Treino 1 & 0 & $\mathrm{O}$ & 1 & 3 & $\mathrm{O}$ & $\mathrm{O}$ & 0 & 4 & 0 & 0 & 1 & 3 \\
\hline Treino 2 & 0 & 0 & 3 & 1 & 0 & 0 & 1 & 3 & 0 & 0 & 1 & 3 \\
\hline Teste 1 & 0 & $\mathrm{O}$ & 0 & 4 & 0 & 0 & 1 & 3 & $\mathrm{O}$ & 0 & 2 & 2 \\
\hline Teste 2 & 0 & 0 & 0 & 4 & 0 & $\mathrm{o}$ & 2 & 2 & 0 & 0 & 1 & 3 \\
\hline Teste 3 & 0 & 0 & 0 & 4 & 0 & 0 & 0 & 4 & 0 & 0 & 1 & 3 \\
\hline $\begin{array}{l}\text { Teste } 4 \\
\text { IVC }\end{array}$ & \multicolumn{4}{|c|}{1} & \multicolumn{4}{|c|}{$0,96^{3}$} & \multicolumn{3}{|c|}{0,96} & 2 \\
\hline
\end{tabular}




\begin{tabular}{|c|c|c|c|c|c|c|c|c|c|c|c|c|}
\hline \multicolumn{13}{|c|}{ Subteste Rima } \\
\hline \multirow{2}{*}{ Rima } & \multicolumn{4}{|c|}{ Compreensão } & \multicolumn{4}{|c|}{ Objetividade } & \multicolumn{4}{|c|}{ Contextualização } \\
\hline & Não & Pouco & Bastante & Muito & Não & Pouco & Bastante & Muito & Não & Pouco & Bastante & Muito \\
\hline Descrição & 0 & 0 & 0 & 4 & 0 & 0 & 0 & 4 & 0 & 0 & 1 & 3 \\
\hline Instruções & 0 & 0 & 0 & 4 & 0 & $\mathrm{o}$ & 0 & 4 & 0 & 0 & 1 & 3 \\
\hline Treino 1 & 0 & 0 & 0 & 4 & 0 & 0 & 0 & 4 & 0 & 0 & o & 4 \\
\hline Treino 2 & 0 & 0 & $\mathrm{O}$ & 4 & $\mathrm{O}$ & $\mathrm{O}$ & $\mathrm{o}$ & 4 & 0 & 0 & 1 & 3 \\
\hline Teste 1 & 0 & 0 & $\mathrm{O}$ & 4 & 0 & 0 & 0 & 4 & $\mathrm{O}$ & 0 & 1 & 3 \\
\hline Teste 2 & 0 & 0 & 0 & 4 & 0 & $\mathrm{o}$ & $\mathrm{O}$ & 4 & 0 & 0 & 0 & 4 \\
\hline Teste 3 & 0 & 0 & 0 & 4 & 0 & 0 & 0 & 4 & 0 & 0 & 1 & 3 \\
\hline $\begin{array}{l}\text { Teste } 4 \\
\text { IVC }\end{array}$ & \multicolumn{4}{|c|}{1} & \multicolumn{4}{|c|}{0,96} & \multicolumn{4}{|c|}{1} \\
\hline \multicolumn{13}{|c|}{ Subteste Aliteração } \\
\hline \multirow{2}{*}{ Aliteração } & \multicolumn{4}{|c|}{ Compreensão } & \multicolumn{4}{|c|}{ Objetividade } & \multicolumn{4}{|c|}{ Contextualização } \\
\hline & Não & Pouco & Bastante & Muito & Não & Pouco & Bastante & Muito & Não & Pouco & Bastante & Muito \\
\hline Descrição & 0 & 0 & 0 & 4 & 0 & 0 & 1 & 3 & 0 & 0 & 2 & 2 \\
\hline Instruções & 0 & 0 & 1 & 3 & o & 0 & 0 & 4 & 0 & 0 & 0 & 4 \\
\hline Treino 1 & 0 & 0 & 0 & 4 & 0 & 0 & 0 & 4 & 0 & 1 & 3 & 0 \\
\hline Treino 2 & 0 & 0 & 1 & 3 & 0 & 0 & 0 & 4 & 0 & 0 & 1 & 3 \\
\hline Teste 1 & 0 & 0 & 0 & 4 & 0 & 0 & 0 & 4 & 0 & 0 & 0 & 4 \\
\hline Teste 2 & $\mathrm{O}$ & 1 & 1 & 2 & 1 & o & o & 3 & 0 & 0 & 0 & 4 \\
\hline Teste 3 & 0 & 0 & 1 & 3 & 0 & 0 & 2 & 2 & 0 & 0 & 1 & 3 \\
\hline $\begin{array}{l}\text { Teste } 4 \\
\text { IVC }\end{array}$ & \multicolumn{4}{|c|}{0,96} & \multicolumn{4}{|c|}{0,96} & \multicolumn{4}{|c|}{0,96} \\
\hline \multicolumn{13}{|c|}{ Subteste Segmentação Silábica } \\
\hline \multirow{2}{*}{ Segmentação Silábica } & \multicolumn{4}{|c|}{ Compreensão } & \multicolumn{4}{|c|}{ Objetividade } & \multicolumn{4}{|c|}{ Contextualização } \\
\hline & Não & Pouco & Bastante & Muito & Não & Pouco & Bastante & Muito & Não & Pouco & Bastante & Muito \\
\hline Descrição & 0 & 0 & 1 & 3 & 0 & 0 & 2 & 2 & 0 & 0 & 3 & 1 \\
\hline Instruções & $\mathrm{o}$ & o & 2 & 2 & $\mathrm{o}$ & $\mathrm{o}$ & 1 & 3 & 0 & 1 & 2 & 1 \\
\hline Treino 1 & o & 0 & 0 & 4 & $\mathrm{o}$ & o & o & 4 & 0 & 0 & 1 & 3 \\
\hline Treino 2 & 0 & $\mathrm{O}$ & 1 & 3 & 0 & 0 & 0 & 4 & 0 & 0 & 1 & 3 \\
\hline Teste 1 & 0 & 0 & 0 & 4 & 0 & 0 & 0 & 4 & 0 & 0 & 1 & 3 \\
\hline Teste 2 & 0 & 0 & 0 & 4 & 0 & 0 & 0 & 4 & 0 & 0 & 0 & 4 \\
\hline Teste 3 & 0 & 0 & $\mathrm{O}$ & 4 & 0 & 0 & 0 & 4 & 0 & 0 & 0 & 4 \\
\hline $\begin{array}{l}\text { Teste } 4 \\
\text { IVC }\end{array}$ & \multicolumn{4}{|c|}{1} & \multicolumn{4}{|c|}{1} & \multicolumn{3}{|c|}{$0,96^{0}$} & 4 \\
\hline
\end{tabular}




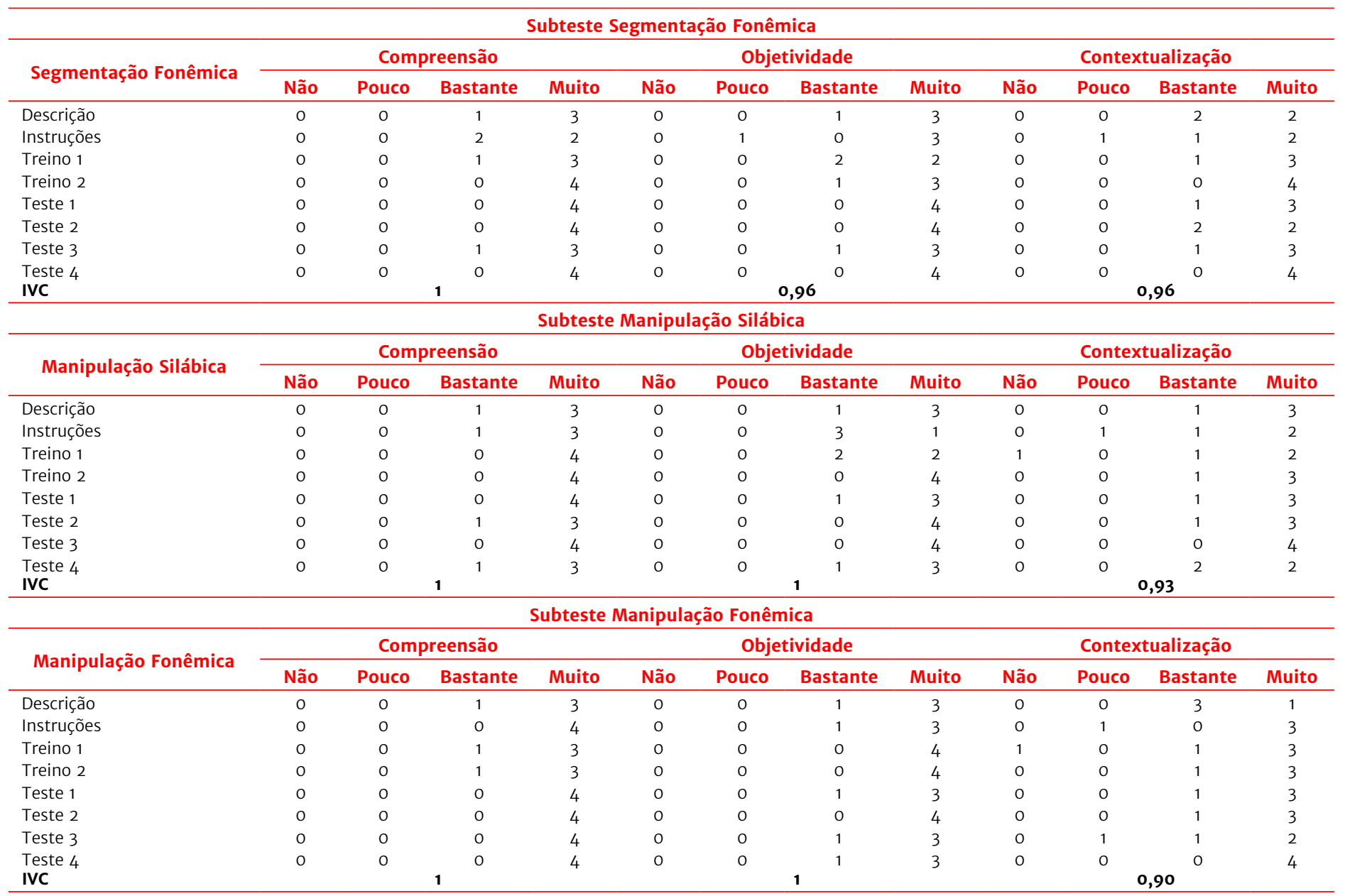




\begin{tabular}{|c|c|c|c|c|c|c|c|c|c|c|c|c|}
\hline \multicolumn{13}{|c|}{ Subteste Transposição Silábica } \\
\hline \multirow{2}{*}{ Transposição Silábica } & \multicolumn{4}{|c|}{ Compreensão } & \multicolumn{4}{|c|}{ Objetividade } & \multicolumn{4}{|c|}{ Contextualização } \\
\hline & Não & Pouco & Bastante & Muito & Não & Pouco & Bastante & Muito & Não & Pouco & Bastante & Muito \\
\hline Descrição & 0 & 0 & 0 & 4 & 0 & 0 & 0 & 4 & 0 & 0 & 3 & 1 \\
\hline Instruções & 0 & 0 & 0 & 4 & 0 & 1 & 1 & 2 & 0 & 1 & 1 & 2 \\
\hline Treino 1 & 0 & 0 & 1 & 3 & 0 & 0 & 1 & 3 & 1 & $\mathrm{o}$ & 2 & 2 \\
\hline Treino 2 & 0 & 0 & 0 & 4 & 0 & 0 & 0 & 4 & 0 & 0 & 2 & 2 \\
\hline Teste 1 & o & 0 & o & 4 & o & o & 1 & 3 & o & 0 & 1 & 3 \\
\hline Teste 2 & o & 0 & 0 & 4 & o & o & 0 & 4 & 0 & 0 & o & 4 \\
\hline Teste 3 & $\mathrm{O}$ & 0 & 0 & 4 & 0 & $\mathrm{O}$ & 1 & 3 & $\mathrm{O}$ & 0 & $\mathrm{O}$ & 4 \\
\hline Teste 4 & 1 & o & o & 3 & 1 & o & 1 & 2 & 1 & 0 & 0 & 3 \\
\hline IVC & \multicolumn{4}{|c|}{0,96} & \multicolumn{4}{|c|}{0,93} & \multicolumn{4}{|c|}{0,90} \\
\hline \multicolumn{13}{|c|}{ Subteste Transposição Fonêmica } \\
\hline \multirow{2}{*}{ Transposição Fonêmica } & \multicolumn{4}{|c|}{ Compreensão } & \multicolumn{4}{|c|}{ Objetividade } & \multicolumn{4}{|c|}{ Contextualização } \\
\hline & Não & Pouco & Bastante & Muito & Não & Pouco & Bastante & Muito & Não & Pouco & Bastante & Muito \\
\hline Descrição & 0 & 0 & 3 & 1 & 0 & 0 & 1 & 3 & 0 & 0 & 2 & 2 \\
\hline Instruções & 0 & 0 & 0 & 4 & 0 & 1 & 2 & 2 & 0 & 1 & 1 & 2 \\
\hline Treino 1 & 0 & 0 & 1 & 3 & 0 & 0 & 1 & 3 & 1 & 0 & 1 & 3 \\
\hline Treino 2 & 0 & 0 & 0 & 4 & 0 & 0 & 0 & 4 & 0 & $\mathrm{O}$ & 1 & 3 \\
\hline Teste 1 & 1 & 0 & 0 & 3 & 1 & 0 & 0 & 3 & 0 & 0 & 1 & 3 \\
\hline Teste 2 & 0 & 0 & 1 & 3 & 0 & 0 & 0 & 4 & 0 & 0 & 0 & 4 \\
\hline Teste 3 & 0 & 0 & 0 & 4 & 0 & 0 & 0 & 4 & 0 & 0 & 0 & 4 \\
\hline Teste 4 & 0 & 0 & 2 & 2 & 0 & 0 & 2 & 2 & \multirow{2}{*}{\multicolumn{4}{|c|}{0,93}} \\
\hline IVC & \multicolumn{4}{|c|}{$0,96^{2}$} & \multicolumn{4}{|c|}{0,93} & & & & \\
\hline \multicolumn{13}{|c|}{ Estrutura e apresentação } \\
\hline \multirow{2}{*}{ Item Adicional } & \multicolumn{4}{|c|}{ Compreensão } & \multicolumn{4}{|c|}{$\begin{array}{l}\text { Objetividade } \\
\end{array}$} & \multicolumn{4}{|c|}{ Contextualização } \\
\hline & Não & Pouco & Bastante & Muito & Não & Pouco & Bastante & Muito & Não & Pouco & Bastante & Muito \\
\hline $\begin{array}{l}\text { Estrutura e apresentação } \\
\text { IVC }\end{array}$ & 0 & 0 & 0 & 4 & 0 & 0 & 1 & 3 & 0 & 0 & 1 & 4 \\
\hline
\end{tabular}

Nota. Informações retiradas do questionário de juízes e especialistas. 
A Tabela 3 apresenta o IVC total da PCFO adaptada, e, desse modo, pode ser verificado que o valor das médias aritméticas de cada seção variou de 0,91 (dados da prova, instruções, aplicação, pontuação) a 1 (estrutura e apresentação).

\section{Tabela 3}

Índice de Validade de Conteúdo (IVC) total da PCFO adaptada

\begin{tabular}{lcccc}
\hline IVC das secções da prova & Compreensão & Objetividade & Contextualização & Média \\
\hline IVC Dados da Prova & 1 & 1 & 0,75 & 0,91 \\
IVC Síntese Silábica & 1 & 1 & 0,96 & 0,98 \\
IVC Síntese Fonêmica & 1 & 0,96 & 0,96 & 0,97 \\
IVC Rima & 1 & 0,96 & 1 & 0,98 \\
IVC Aliteração & 0,96 & 0,96 & 0,96 & 0,96 \\
IVC Segmentação Silábica & 1 & 1 & 0,96 & 0,98 \\
IVC Segmentação Fonêmica & 1 & 0,96 & 0,96 & 0,97 \\
IVC Manipulação Silábica & 1 & 1 & 0,93 & 0,97 \\
IVC Manipulação Fonêmica & 1 & 1 & 0,90 & 0,96 \\
IVC Transposição Silábica & 0,96 & 0,93 & 0,90 & 0,93 \\
IVC Transposição Fonêmica & 0,96 & 0,93 & 0,93 & 0,94 \\
IVC Estrutura e apresentação & 1 & 1 & 1 & 1 \\
IVC TOTAL & $\mathbf{0 , 9 9}$ & $\mathbf{0 , 9 7}$ & $\mathbf{0 , 9 3}$ & $\mathbf{0 , 9 6}$ \\
\hline
\end{tabular}

Nota. Informações retiradas do questionário de juízes e especialistas.

Além disso, os juízes também sugeriram mudanças no instrumento, e a Tabela 4 apresenta as adaptações que realizaram na PCFO preliminar.

\section{Tabela 4}

Adaptações realizadas por juízes na PCFO preliminar

\begin{tabular}{|c|c|c|c|c|}
\hline Prova & Item & $\begin{array}{c}\text { Palavra/expressão da } \\
\text { PCFO original }\end{array}$ & Juízes & $\begin{array}{l}\text { Adaptação } \\
\text { realizada }\end{array}$ \\
\hline Dados da Prova & Dados da prova & $\begin{array}{l}\text { "...Pontuação 1/2 pode } \\
\text { ser atribuída } \\
\text { em casos..." }\end{array}$ & 3 & $\begin{array}{l}\text { "...Metade da } \\
\text { pontuação }(0,5) \\
\text { pode ser atribuída } \\
\text { nos casos..." }\end{array}$ \\
\hline \multirow{2}{*}{$\begin{array}{l}\text { Síntese Silábica } \\
\text { e Fonêmica }\end{array}$} & Descrição & $\begin{array}{l}\text { "...dizendo qual palavra } \\
\text { resulta da união." }\end{array}$ & 2 & $\begin{array}{l}\text { "...e dizer qual é a } \\
\text { palavra que resulta } \\
\text { dessa união." }\end{array}$ \\
\hline & Instrução & $\begin{array}{l}\text { "...e você deve } \\
\text { adivinhar..." }\end{array}$ & 2 & $\begin{array}{l}\text { "...e a sua tarefa é } \\
\text { de adivinhar..." }\end{array}$ \\
\hline Síntese Fonêmica & Treino 2 & Laço & 1 & Pato \\
\hline
\end{tabular}




\begin{tabular}{|c|c|c|c|c|}
\hline Prova & Item & $\begin{array}{c}\text { Palavra/expressão da } \\
\text { PCFO original }\end{array}$ & Juízes & $\begin{array}{c}\text { Adaptação } \\
\text { realizada }\end{array}$ \\
\hline \multirow{3}{*}{ Rima } & Teste 3 & Folha & 1 & Filha \\
\hline & & Bolha & 1 & Pilha \\
\hline & Teste 4 & Maré & 1 & Sopé \\
\hline Aliteração & Treino 1 & Face & 1 & Faca \\
\hline $\begin{array}{l}\text { Segmentação } \\
\text { Silábica e Fonêmica }\end{array}$ & Instruções & $\begin{array}{l}\text { "...o robô, repetindo a } \\
\text { palavra..." }\end{array}$ & 2 & $\begin{array}{l}\text { "...o robô, a sua } \\
\text { tarefa é de repetir } \\
\text { a palavra..." }\end{array}$ \\
\hline $\begin{array}{l}\text { Segmentação } \\
\text { Fonêmica }\end{array}$ & Instrução & $\begin{array}{l}\text { “..., mas agora falando } \\
\text { as partes menores..., } \\
\text { falando cada som } \\
\text { separadamente." }\end{array}$ & 2 & $\begin{array}{l}\text { "..., mas agora vai } \\
\text { falar as partes } \\
\text { menores..., isto é, } \\
\text { cada som } \\
\text { separadamente." }\end{array}$ \\
\hline \multirow[t]{2}{*}{$\begin{array}{l}\text { Manipulação } \\
\text { Silábica e Fonêmica }\end{array}$} & Descrição & $\begin{array}{l}\text { "...sílabas de palavras } \\
\text { dizendo qual a } \\
\text { palavra..." }\end{array}$ & 2 & $\begin{array}{l}\text { "...sílabas de } \\
\text { palavras e dizer } \\
\text { qual é a palavra..." }\end{array}$ \\
\hline & Instrução & $\begin{array}{l}\text { "Você vai dizer } \\
\text { como fica..." }\end{array}$ & 2 & $\begin{array}{l}\text { "A sua tarefa é } \\
\text { dizer como fica..." }\end{array}$ \\
\hline $\begin{array}{l}\text { Manipulação } \\
\text { Silábica }\end{array}$ & Treino 1 & Macarrão & 1 & Camarão \\
\hline $\begin{array}{l}\text { Manipulação } \\
\text { Fonêmica }\end{array}$ & Teste 3 & Louça & 1 & Couve \\
\hline \multirow{3}{*}{$\begin{array}{l}\text { Transposição } \\
\text { Silábica e Fonêmica }\end{array}$} & Descrição & $\begin{array}{l}\text { "...dizendo qual a } \\
\text { palavra formada." }\end{array}$ & 2 & $\begin{array}{l}\text { "...e dizer qual é a } \\
\text { palavra formada." }\end{array}$ \\
\hline & & & & \\
\hline & Instruções & $\begin{array}{l}\text { "Você vai falar uma } \\
\text { palavra..." }\end{array}$ & 2 & $\begin{array}{l}\text { "A sua tarefa é } \\
\text { falar uma } \\
\text { palavra..." }\end{array}$ \\
\hline $\begin{array}{l}\text { Transposição } \\
\text { Silábica }\end{array}$ & & Partes & 1 & Sílaba \\
\hline
\end{tabular}

Nota. Dados do questionário de validação realizada por juízes e especialistas.

\section{Discussão}

Este estudo teve por objetivo adaptar um instrumento brasileiro para o contexto moçambicano. Um dos critérios a ser considerado para escolha de um instrumento para ser adaptado é que ele possua requisitos básicos, tais como evidências de confiabilidade e validade, para além de fornecer resultados cientificamente robustos nos seus estudos. Esse aspecto foi levado em consideração, e verificou-se que a PCFO é um instrumento que vem sendo usado em pesquisas com a população brasileira nos últimos 14 anos, possuindo estudos de validade e precisão com uso de técnicas diversificadas (Dias et al., 2013)

Em relação à adaptação propriamente dita, pesquisas sugerem que esse processo pode levar a resultados duvidosos se feito de maneira inadequada do ponto de vista linguístico, em 
relação às expressões comuns de um determinado contexto. Alguns estudos mostraram a importância da adaptação semântica durante o processo de desenvolvimento ou adaptação de instrumentos, embora a metodologia usada neles não tenha sido por meio de nativos, tal como foi usada aqui. Por exemplo, Freitas et al. (2001), em um estudo de tradução e adaptação, observaram que, mesmo depois de equivalência conceitual e ser preservado o layout do instrumento original, as maiores dificuldades na tradução estavam relacionadas à equivalência semântica, no que concerne às formas coloquiais e expressões idiomáticas do português traduzidas a partir do instrumento original. Contudo, no fim do processo, os problemas foram resolvidos por consenso entre os autores, muitos deles priorizando a adaptação à cultura brasileira, em detrimento da equivalência semântica dos especialistas. Num outro estudo, King e Bhugra (1989), que avaliaram populações subdesenvolvidas e não ocidentais na Índia e no Paquistão, usaram um instrumento traduzido por especialistas, contudo, na análise dos dados, verificaram que havia padrões consistentes de respostas em cinco itens que, segundo eles, poderiam ter sido devido a problemas socioculturais e interpretações, o que levou os autores ao uso de expressões e conceitos que tivessem significado adequado para a população por conta de influências linguísticas, sociais e religiosas.

No que diz respeito à validação por especialistas, é uma etapa muito importante e que tem sido apresentada em muitos estudos de tradução e adaptação cultural de instrumentos. Por exemplo, em um estudo português que tinha como objetivos desenvolver e validar um instrumento para medição da qualidade de vida para doentes em cuidados paliativos, Ferreira e Pinto (2008), por meio do teste de compreensão e validação de conteúdo, identificaram que os pacientes apresentavam dúvidas e hesitações quanto ao número de respostas que poderiam marcar numa determinada pergunta, o que depois foi considerado pelos autores e ajudou a melhorar a qualidade da versão portuguesa do instrumento. Num outro estudo que estava em andamento, em que o instrumento a ser aplicado foi desenvolvido e não possuía validação semântica, Bausewein et al. (2005) questionaram se a redação da versão alemã era clara e compreensível. Realizadas as entrevistas com os participantes, os resultados mostraram que $7 \%$ gostaram muito, $59 \%$ gostaram, $29 \%$ ficaram indecisos e $5 \%$ não gostaram muito da escala. Deve-se lembrar que todos os estudos indicados anteriormente consideravam que as versões da escala produzidas apresentavam bons níveis de confiabilidade e outros indicadores aceitos para sua utilização. Assim, o reconhecimento da qualidade dos instrumentos torna-se um aspecto fundamental para a legitimidade e credibilidade dos resultados de uma pesquisa (Medeiros et al., 2015).

Em relação ao IVC, há uma discussão entre pesquisadores sobre o seu valor, sendo necessária, em alguns casos, uma concordância de pelo menos $80 \%$ entre os juízes para servir de critério para aceitação do instrumento (Pasquali, 2010) e, em outros, concordância maior ou igual a 90\% (Wynd et al., 2003). Para este estudo, o IVC total de todo instrumento foi de 
0,96 (96\%), percentual de concordância dos juízes que também foi encontrado no estudo de Pieri et al. (2017). Tanto na primeira quanto na segunda abordagem sobre o valor do IVC, a PCFO adaptada apresentou um ótimo IVC que garante que o instrumento possa ser aplicado para a população moçambicana. Mesmo assim, podem ser discutidos esses achados e feitas algumas inferências sobre ele, uma vez que o valor do IVC ficou tão próximo de 1, ou seja, 100\% de concordância.

A primeira explicação pode dar-se porque o instrumento passou por um processo de adequação semântica realizada por nativos moçambicanos residentes no país de origem do instrumento, o que provavelmente facilitou que os nativos fizessem algumas adequações por conhecerem muitas expressões brasileiras. A segunda pode ser em decorrência do número dos juízes, uma vez que, na literatura, estudos sugerem que naqueles em que participa um mínimo de cinco avaliadores a taxa tem sido de no mínimo $90 \%$ de concordância (Lynn, 1986). A terceira e última pode ser porque o processo de adaptação cultural do instrumento envolveu dois países ou contextos que falam o mesmo idioma, o que teoricamente não precisaria de muitos ajustes se comparado a um processo de tradução e adaptação de um instrumento, cujo idioma fosse diferente entre os dois contextos, ou provavelmente se fosse realizado a partir de uma variante do português que não compartilhasse as mesmas variedades. Segundo Petter (2008), o vocabulário básico, constituído majoritariamente de termos de origem portuguesa, é compartilhado pelas variedades angolana, brasileira e moçambicana de português. De acordo com a mesma autora, esse vocabulário apresenta alguns sinônimos locais e manifesta um uso exclusivo não compartilhado com o português europeu, além de apresentar, com relativa autonomia, o uso dos processos de criação lexical do português europeu.

Entretanto, a utilização de nativos foi de bastante relevância por tratar-se de uma das fases metodológicas fundamentais em estudos de adaptação cultural de instrumentos (Hambleton \& Zenisky, 2010). Especificamente neste estudo, o uso de nativos residentes no país de origem do instrumento teve por finalidade facilitar a análise e o julgamento do instrumento na fase 2. Isso ocorreu porque os juízes provavelmente teriam mais dificuldades na compreensão de muitos vernáculos usados exclusivamente no português brasileiro caso o instrumento não fosse adaptado anteriormente ou se fosse adaptado por nativos que nunca tiveram contato com o português e com a cultura brasileira. Todos os aspectos mencionados anteriormente podem ter deixado o processo de adaptação como um todo mais viável e menos exigente. Além disso, o julgamento dos especialistas foi mais claro e objetivo, o que se verificou, em partes, pelas poucas adaptações realizadas na PCFO e consequentemente contribuiu para a obtenção do valor alto do IVC.

No que diz respeito aos valores do IVC de cada seção, verificou-se que a pontuação mais baixa $(0,75)$ foi no critério "contextualização" da seção "dados da prova". Nesse caso em 
particular, não foram problemas de contextualização ou no número de adaptações e sugestões dos especialistas que influenciaram essa pontuação. Provavelmente, isso se deu devido ao número de itens (um) avaliados nessa seção, em que o primeiro juiz pontuou como item pouco claro, o que consequentemente acabou influenciando no cálculo e nesse resultado em particular. Por sua vez, a seção "estrutura e apresentação da prova" foi a única que teve 100\% de concordância entre os juízes. Esse fato pode ser devido à ideia de que, na medida do possível, a apresentação do instrumento adaptado pode parecer igual ou muito semelhante em todas as versões de idiomas. Por exemplo, O'Connor e Malak (2000) referem que as pesquisas sugerem que, na apresentação de versões traduzidas de instrumentos, os itens devem permanecer no mesmo padrão, ordem e local da página em todas as versões. Assim, talvez, em razão desse cuidado dos juízes, esse item do instrumento adaptado tivesse $100 \%$ de concordância.

Concluindo, verificou-se que, embora a PCFO tenha sido desenvolvida em língua portuguesa (mesmo idioma oficial também em Moçambique), havia necessidade de ajustar os itens da prova para vernáculos do português falado em Moçambique. Todo o processo foi realizado de forma cuidadosa, sistematizada e satisfatória, respeitando os valores socioculturais dos dois contextos, tendo se cumprido assim com os objetivos propostos. Não houve eliminação de nenhum subteste da versão original, ainda que tenham sido realizadas pequenas adequações devido a diferenças dos idiomas dos dois contextos.

Contudo, embora a PCFO adaptada tenha apresentado ótimas evidências de validade de conteúdo, mais estudos de validade e precisão para além de dados normativos devem ser realizados nos estudos futuros, visando garantir a qualidade do instrumento para o uso na clínica e em pesquisas científicas. Além desse fato, algumas limitações do estudo precisam ser consideradas. A primeira refere-se ao fato de que essa etapa de validação é considerada subjetiva por envolver opiniões de especialistas, fazendo com que outras evidências de validade e precisão sejam realizadas nos estudos futuros. A segunda está relacionada ao fato de que não houve interação entre os membros do comitê de juízes para que pudessem chegar a uma posição consensual das adaptações. A terceira tem a ver com a não realização de um controle em relação aos componentes ou com a composição fonológica das palavras adaptadas, sugerindo-se assim que nos próximos estudos seja controlado esse aspecto fundamental. A quarta e última está relacionada à pouca literatura existente para estudos de adaptação cultural, em que o contexto de origem do instrumento e o contexto de destino falam o mesmo idioma, embora a literatura aponte a importância e necessidade desses estudos, principalmente em casos de população imigrante morando num determinado contexto.

Apesar das limitações, espera-se que todo o processo de adaptação seja uma etapa inicial e fundamental, cujo objetivo final vai para além de disponibilizar o instrumento para a realidade moçambicana. Para o setor de educação especificamente, este estudo é muitíssimo 
relevante, principalmente para avaliação e estimulação de habilidades consideradas fundamentais para o desempenho acadêmico e intervenção destinadas a elas. Espera-se que, com a existência do instrumento, haja uma reflexão e um debate profundos, de todas as frentes envolvidas no setor, sobre a importância da habilidade. Com a difusão desse conhecimento, há a expectativa de que gradativamente sejam introduzidas atividades lúdicas e curriculares, contemplando a CF nos serviços de primeira infância e principalmente em creches e jardins de infância, para além de que mais estudos da área sejam conduzidos, mostrando a importância da habilidade para crianças antes da entrada no ensino formal. 


\section{Referências}

Bausewein, C., Fegg, M., Radbruch, L., Nauck, F., Von Mackensen, S., Borasio, G. D., \& Higginson, I. J. (2005). Validation and clinical application of the german version of the palliative care outcome scale. Journal of Pain and Symptom Management, 30, 51-62. https://doi.org/10.1016/j. jpainsymman.2005.01.017

Capovilla, A. G. S., \& Capovilla, F. C. (1998). Prova de consciência fonológica: Desenvolvimento de dez habilidades da pré-escola à segunda série. Temas Sobre Desenvolvimento, 7, 14-20.

Capovilla, A. G. S., Dias, N. M., \& Montiel, J. M. (2007). Desenvolvimento dos componentes da consciência fonológica no ensino fundamental e correlação com nota escolar. Psico-USF, 12, 55-64. https://doi.org/10.1590/s1413-82712007000100007

Ciesielski, E. J. M., \& Creaghead, N. A. (2020). The effectiveness of professional development on the phonological awareness outcomes of preschool children: A systematic review. Literacy Research and Instruction, 59, 121-147. https://doi.org/10.1080/19388071.2019.1710785

Coluci, M. Z. O., Alexandre, N. M. C., \& Milani, D. (2015). Construção de instrumentos de medida na área da saúde. Ciência e Saúde Coletiva, 20, 925-936. https://doi.org/10.1590/ $1413-81232015203.04332013$

Diamanti, V., Mouzaki, A., Ralli, A., \& Antoniou, F. (2017). Preschool phonological and morphological awareness as longitudinal predictors of early reading and spelling development in Greek. Frontiers in Psychology, 8, 1-12. https://doi.org/10.3389/fpsyg.2017.02039

Dias, N. M., Duarte, C. P., Seabra, A. G., \& Macedo, E. C. de. (2013). Evidências de validade e fidedignidade da Prova de Consciência Fonológica por produção Oral. In A. G. Seabra \& N. M. Dias (Eds.), Avaliação Neuropsicológica Cognitiva: Linguagem oral (2nd ed., pp. 110-116). Memnon.

Dias, N. M., Trevisan, B. T., \& Seabra, A. G. (2013). Dados normativos da Prova de Consciência Fonológica por produção Oral. In A. G. Seabra \& N. M. Dias (Eds.), Avaliação Neuropsicológica Cognitiva: Linguagem oral (2nd ed., pp. 109-115). Memnon.

Fälth, L., Gustafson, S., \& Svensson, I. (2017). Phonological awareness training with articulation promotes early reading development. Education (Chula Vista, CA), 137, 261-276. https://doi.org/ https://www.researchgate.net/publication/314758638

Ferreira, P., \& Pinto, A. (2008). Medir qualidade de vida em cuidados paliativos. Acta Med Port., 21, 111-124.

Fonseca, R. P., Casarin, F. S., De Oliveira, C. R., Gindri, G., Ishigaki, E. C. S. S., Ortiz, K. Z., Parente, M. A. D. M. P., \& Scherer, L. C. (2011). Adaptação de instrumentos neuropsicológicos verbais: Um fluxograma de procedimentos para além da tradução. Interação Em Psicologia, 15, 59-69. https://doi.org/10.5380/psi.v15io.25374

Francisco, A. (2015). Estudo exploratório da situação da educação nos países participantes no PCSS-Lusófonos - Relatório de Moçambique (No. 2). 1-138.

Freitas, S., Lopes, C. S., Coutinho, W., \& Appolinario, J. C. (2001). Tradução e adaptação para o português da Escala de Compulsão Alimentar Periódica. Rev Bras Psiquiatr, 23, 215-220.

Fundação Aga Khan. (2011). Uso efectivo do tempo nas escolas de Cabo Delgado. Aga Khan. 
Germano, G. D., Pinheiro, F. H., \& Capellini, S. A. (2013). Desempenho de escolares com dislexia: Programas de intervenção metalinguístico e de leitura. Psicol. Argum. Curitiba, 31, 11-22.

Goswami, U., \& Bryant, P. E. (1900). Phonological skills and learning to read. Psychology Press.

Grant, J. S., \& Davis, L. L. (1997). Selection and use of content experts for instrument development. Research in Nursing \& Health, 20, 269-274. https://doi.org/10.1002/ (SICI)1098-240X(199706)20:3<269::AID-NUR9>3.0.CO;2-G

Guillemin, F. (1995). Cross-cultural adaptation and validation of health status measures. Scandinavian Journal of Rheumatology, 24, 61-63. https://doi.org/10.3109/03009749509099285

Hambleton, R. K. (2005). Issues, designs, and technical guidelines for adapting tests into multiple languages and cultures. In R. K. Hambleton, P. F. Merenda, \& C. D. Spielberger (Eds.), Adapting Educational and Psychological Tests for Cross-Cultural Assessment (pp. 3-38). Lawrence Erlbaum Associates.

Hambleton, R. K., \& Zenisky, A. L. (2010). Translating and Adapting Tests for Cross-Cultural Assessments. In D. Matsumoto \& F. J. R. van de Vijver (Eds.), Cross-Cultural Research Methods in Psychology (pp. 46-70). Cambridge University Press. https://doi.org/10.1017/CBO9780511779381.004

King, M. B., \& Bhugra, D. (1989). Eating disorders: Lessons from a cross-cultural study. Psychological Medicine, 19, 955-958. https://doi.org/10.1017/S0033291700005675

Kjeldsen, A. C., Educ, L., Saarento-Zaprudin, S. K., \& Niemi, P. O. (2019). Kindergarten training in phonological awareness: Fluency and comprehension gains are greatest for readers at risk in grades 1 through 9. Journal of Learning Disabilities, 52, 366-382. https://doi. org/10.1177/0022219419847154

Lynn, M. R. (1986). Determination and Quantification of Content Validity. Journal of Experimental Psychology, 136, 382-386. https://doi.org/10.1097/00006199-198611000-00017

Medeiros, R. K. da S., Júnior, M. A. F., Pinto, D. P. D. S. R., Vitor, A. F., Santos, V. E. P., \& Barichello, E. (2015). Modelo de validação de conteúdo de Pasquali nas pesquisas em Enfermagem. Revista de Enfermagem Referência, 4, 127-135. https://doi.org/10.12707/RIV14009

Morais, J. G. O. de. (2019). Geopolítica e História Econômica das relações Brasil-Moçambique: A longa duração e a inflexão no governo Lula [Unpublished Master's Dissertation]. Universidade de São Paulo. https://doi.org/10.11606/D.31.2020.tde-16012020-124421

Nhampossa, C. D. J. (2018). Projecto "Turma Mais": Proposta de combate ao insucesso escolar na Matemática no Ensino Básico Moçambicano. UDZIWI, Revista de Educação Da Universidade Pedagógica, 30, 42-59.

O'Connor, K. M., \& Malak, B. (2000). Translation and cultural adaptation of the TIMSS instruments. In M. O. Martin, K. D. Gregory, \& S. E. Stemler (Eds.), TIMSS 1999 technical report (pp. 89-100). Chestnut Hill.

Pape-neumann, J., Ermingen-marbach, M. Van, Grande, M., \& Willmes, K. (2015). The role of phonological awareness in treatments of dyslexic primary school children. Acta Neurobiol Exp, $75,80-106$.

Pasquali, L. (2003). Psicometria: Teoria dos testes na psicologia e na educação (5a ed.). Editora Vozes.

Pasquali, L. (2010). Instrumentação psicológica: Fundamentos e práticas. Artmed. 
Pasquali, L. (2013). Psicometria: Teoria dos testes na psicologia e na educação (5 ed.). Editora Vozes.

Pazeto, T. de C. B., Dias, N. M., Gomes, C. M. A., \& Seabra, A. G. (2020). Prediction of arithmetic competence: Role of cognitive abilities, socioeconomic variables and the perception of the teacher in Early Childhood Education. Estudos de Psicologia, 24, 225-236. https://doi. org/10.22491/1678-4669.20190024

Petter, M. (2008). O léxico compartilhado pelo português angolano, brasileiro e moçambicano. VEREDAS (Revista Da Associação Internacional de Lusitanistas), 9, 61-81.

Petter, M. M. T. (2009). Aspectos morfossintáticos comuns ao português angolano, brasileiro e moçambicano. PAPIA - Revista Brasileira de Estudos Crioulos e Similares, 19, 201-220.

Pieri, R. D. V., Pires, P., Filgueiras, A., \& Oliva, Â. D. (2017). Adaptação Transcultural e Validação de Conteúdo do Questionário de Coesão no Esporte Infantil para o Português do Brasil. Revista Brasileira de Psicologia Do Esporte, 6, 14-25. https://doi.org/10.31501/rbpe.v6i2.7088

Pinto, G., Bigozzi, L., Tarchi, C., Vezzani, C., \& Gamannossi, B. A. (2016). Predicting reading, spelling, and mathematical skills: A longitudinal study from kindergarten through first grade. Psychological Reports, 118, 413-440. https://doi.org/10.1177/0033294116633357

Santos, A. A. A. dos, Ferraz, A. S., Lima, T. H. de, Cunha, N. de B., Suehiro, A. C. B., Oliveira, K. L. de, Anache, A. A., \& Silva, I. R. da. (2017). Habilidades linguísticas: A relação entre a consciência fonológica e a escrita. Estud. Pesqui. Psicol., 17, 575-594.

Seabra, A. G., \& Capovilla, F. C. (2011). Problemas de leitura e escrita: Como identificar, prevenir e remediar numa abordagem fônica ( 6 ed.). Memnon.

Seabra, A. G, \& Capovilla, F. C. (2012). Prova de Consciência Fonológica por Produção Oral Linguagem oral. In A. G Seabra \& N. M. Dias (Eds.), Avaliação Neuropsicológica Cognitiva. Memnon.

Shanahan, T., \& Lonigan, C. J. (2015). The national early literacy panel: A summary of the process and the report. Educational Researcher, 39, 279-285. https://doi.org/10.3102/0013189X10369172

Snowling, M. J., \& Melby-Lervåg, M. (2016). Oral language deficits in familial dyslexia: A meta-analysis and review. Psychological Bulletin, 142, 498-545. https://doi.org/10.1037/buloooo037

Song, S., Georgiou, G. K., Su, M., \& Hua, S. (2015). Scientific Studies of Reading How well do phonological awareness and rapid automatized naming correlate with Chinese reading accuracy and fluency? A meta-analysis. Scientific Studies of Reading, 8438. https://doi.org/10.1080/10888438. 2015.1088543

Timbane, A. A. (2016). O ensino da língua portuguesa em Moçambique e a problemática da formação de professores primários. Revista Do Difere, 4, 1-21.

TPC Moçambique. (2017). Será que as nossas crianças estão a aprender? Relatório anual sobre a aprendizagem em Moçambique (fase piloto, Nampula, 2016) (A. Ali (ed.). Elográfico.

UNESCO. (2007). Relatório de Monitoramento Global de Educação Para Todos - EPT 2007 - Bases Sólidas: Educação e Cuidados na Primeira Infância. UNESCO.

UNESCO. (2012). Expanding equitable early childhood care and education is an urgent need. Education for All Global (3rd ed.). UNESCO.

Vibulpatanavong, K., \& Evans, D. (2019). Phonological awareness and reading in Thai children. Reading and Writing, 32, 467-491. https://doi.org/10.1007/s11145-018-9867-0 
Wynd, C. A., Schmidt, B., \& Schaefer, M. A. (2003). Two quantitative approaches for estimating content validity. Western Journal of Nursing Research, 25, 508-518.

\section{EQUIPE EDITORIAL}

Editora-chefe

Cristiane Silvestre de Paula

\section{Editores associados}

Alessandra Gotuzo Seabra Ana Alexandra Caldas Osório Luiz Renato Rodrigues Carreiro Maria Cristina Triguero Veloz Teixeira

\section{Editores de seção}

"Avaliação Psicológica"

Alexandre Luiz de Oliveira Serpa

André Luiz de Carvalho Braule Pinto

Luiz Renato Rodrigues Carreiro Vera Lúcia Esteves Mateus

\section{"Psicologia e Educação"}

Alessandra Gotuzo Seabra

Carlo Schmidt

Regina Basso Zanon
"Psicologia Social e Saúde das Populações"

Enzo Banti Bissoli

Marina Xavier Carpena

\section{"Psicologia Clínica"}

Carolina Andrea Ziebold Jorquera

Julia Garcia Durand

Natalia Becker

\section{"Desenvolvimento Humano"}

Maria Cristina Triguero

Veloz Teixeira

Rosane Lowenthal

Suporte técnico

Maria Fernanda Liuti

Bento da Silva

Camila Fragoso Ribeiro
PRODUÇÃO EDITORIAL

Coordenação editorial

Ana Claudia de Mauro

Estagiários editoriais

Júlia Lins Reis

Pietro Menezes

Élcio Carvalho

Preparação de originais

Carlos Villarruel

\section{Revisão}

Vera Ayres

Diagramação

Acqua Estúdio Gráfico 